\title{
Indoor localization: novel RSSI approach based on analytical solution and two receivers
}

\author{
Ahmad Warda ${ }^{1}$, Bojana Petković ${ }^{2}$, and Hannes Töpfer ${ }^{1}$ \\ ${ }^{1}$ Institute for Information Technology, Technische Universität Ilmenau, Ilmenau, Germany \\ ${ }^{2}$ Institute of Biomedical Engineering and Informatics, Technische Universität Ilmenau, Ilmenau, Germany \\ Correspondence to: Ahmad Warda (ahmad.warda@tu-ilmenau.de)
}

Received: 26 June 2017 - Revised: 23 August 2017 - Accepted: 10 October 2017 - Published: 20 November 2017

\begin{abstract}
Indoor localization based on trilateration method uses at least three receivers for an accurate localization in 2-D. We performed indoor localization in 2-D using only two receivers, combining algebraic equations for signal strengths into one quadratic equation with transmitter position as unknown and using a specific receiver placement at the two adjacent corners of the room. This receiver arrangement assures unique coordinates of the transmitter position inside a room, rejecting automatically the other solution which appears outside the room volume. The accuracy of the method is numerically tested in a room with dimensions of $9.7 \mathrm{~m} \times 4.7 \mathrm{~m} \times 3 \mathrm{~m}$ and shows a mean reconstruction error of $3.4 \mathrm{~cm}$.
\end{abstract}

\section{Introduction}

Received signal strength indicator (RSSI), defined by IEEE 802.11 standards, is the most widely used signal feature in wireless localization systems. It is especially suitable for wireless local area network (WLAN) because of an easy extraction in 802.11 with a capability of running on off-shelf WLAN hardware. RSSI is the measured radio-frequency energy in units of dBm (Farid et al., 2013; Yassin et al., 2016). An accurate and unique RSSI-based localization is achieved using a trilateration with at least three non-on-one-line receivers in 2-D and at least four receivers in 3-D (Zhang et al., 2009). In the study by Warda et al. (2017), we introduced the scanning method based on fingerprint to overcome the fingerprint drawbacks, and we compared its performance with fingerprint and trilateration for localization of a mobile robot with three receivers in 2-D. Localization could also be performed using two receivers; however, there is a problem of the existence of two solutions and ambiguity of a correct one (Linde, 2006). This problem has overcome incorporating a priori information about the approximate position of the target (Fang, 1990). Placement of receivers affects substantially the performance of the localization algorithm (Bishop et al., 2010), who found that the optimal placement of two receivers in range-based localization is when they form an angle with the target of $\frac{\pi}{2}$. For three receivers, there are two optimal placement possibilities according to corollary 1 in Bishop et al. (2010), $\frac{\pi}{3}$, and $\frac{2 \pi}{3}$. Han et al. (2007) proposed improving localization accuracy, placing the receivers on vertices of equilateral triangles. Based on such a placement, they introduced an algorithm for localization which, according to their study, outperforms the random and square-grid placement in time and accuracy.

In this paper, we introduce a new source localization scheme based on quadratic equation approach and using only two receivers placed at the corners of the ceiling of the room. Such a recommendation for sensor arrangement could easily be followed in every room very quickly. Furthermore, this arrangement assures that one of the roots of quadratic equation is outside the room volume, provided that the required transmitter position is unique. We briefly describe the method in Sect. 2, show the simulation results in Sect. 3, and derive the conclusions in Sect. 4.

\section{Methods}

Considering two receivers, their placement, and a forward model, a quadratic equation will be formulated and solved. The localization task will be exemplified using transceivers commonly used in wireless sensor networks (WSNs). The target of localization or tracking scenario can be a person or a mobile robot equipped with a transceiver. 


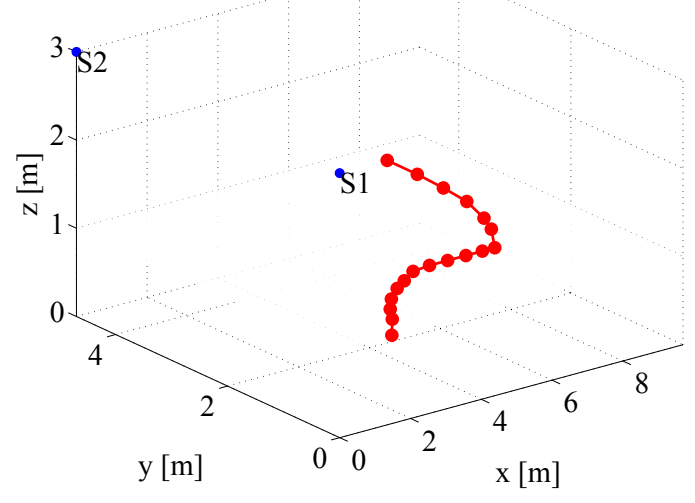

Figure 1. Two receivers are placed at $3 \mathrm{~m}$ in height and presented by blue points. The path of the moving transmitter ( 18 positions) is presented by red points, joined by a red solid line.

\section{Analytical solution}

The received power $P_{\mathrm{r}}$ at the position of the receiver $\boldsymbol{R}_{i}$ is related to the transmitted power $P_{\mathrm{t}}$ at the position $\boldsymbol{r}_{j}$ according to the forward model (Eq. 1). It has been assumed in this equation that there is a direct path between the receiver and the transmitter and no signal interference occurrence (Garg, 2007):

$P_{\mathrm{r}}=P_{\mathrm{t}}\left(\frac{\lambda}{4 \pi r}\right)^{2} G_{\mathrm{r}} G_{\mathrm{t}}$,

where $r=\left|\boldsymbol{R}_{\boldsymbol{i}}-\boldsymbol{r}_{j}\right|$ represents the distance between the transmitter and the receiver, $\lambda$ is the wavelength of propagation, and $G_{\mathrm{r}}$ and $G_{\mathrm{t}}$ are the gains of the receiver and the transmitter antenna, respectively.

Considering that all parameters in Eq. (1) are constants except the distance, we can write this equation in the following form:

$P_{\mathrm{r}}=\frac{c_{1}}{r^{2}}$

where $c_{1}=P_{\mathrm{t}} G_{\mathrm{r}} G_{\mathrm{t}} \lambda^{2} /(4 \pi)^{2}$. Using Eq. (2), the received power $P_{\mathrm{r}_{1}}$ of the first receiver at the position $\boldsymbol{R}_{1}$ and the received power $P_{\mathrm{r}_{2}}$ of the second receiver at the position $\boldsymbol{R}_{2}$ can be written as follows:

$P_{\mathrm{r}_{1}}=\frac{c_{1}}{r_{1}^{2}}=\frac{c_{1}}{\left(x_{\mathrm{s} 1}-x_{\mathrm{t}}\right)^{2}+\left(y_{\mathrm{s} 1}-y_{\mathrm{t}}\right)^{2}+\left(z_{\mathrm{s} 1}-z_{\mathrm{t}}\right)^{2}}$,

$P_{\mathrm{r}_{2}}=\frac{c_{1}}{r_{2}^{2}}=\frac{c_{1}}{\left(x_{\mathrm{s} 2}-x_{\mathrm{t}}\right)^{2}+\left(y_{\mathrm{s} 2}-y_{\mathrm{t}}\right)^{2}+\left(z_{\mathrm{s} 2}-z_{\mathrm{t}}\right)^{2}}$,

where $x_{\mathrm{s} 1}, y_{\mathrm{s} 1}$, and $z_{\mathrm{s} 1}$ are the Cartesian coordinates of receiver $\mathrm{S} 1 ; x_{\mathrm{s} 2}, y_{\mathrm{s} 2}$, and $z_{\mathrm{s} 2}$ are the coordinates of receiver $\mathrm{S} 2$; and $x_{\mathrm{t}}, y_{\mathrm{t}}$, and $z_{\mathrm{t}}$ are unknown coordinates of the transmitter. By subtracting and reordering (see Appendix A), we get

$a x_{\mathrm{t}}^{2}+b x_{\mathrm{t}}+c=0$.

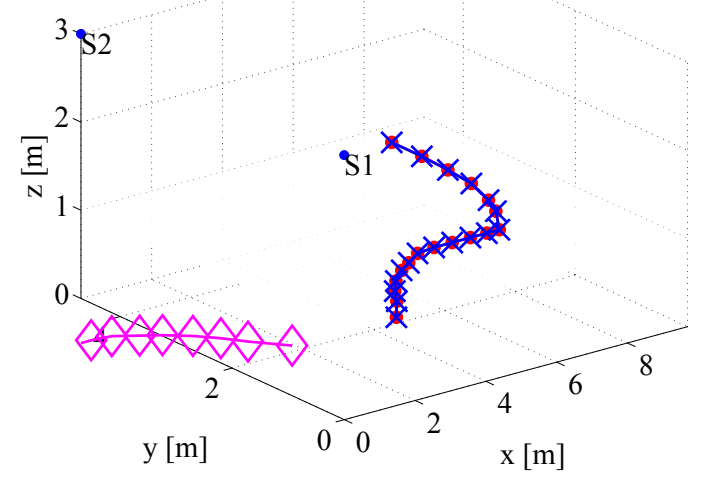

Figure 2. Simulation I: reconstruction of the path when receivers $\mathrm{S} 1$ and S2 are located along the $y$ axis at the corners of the ceiling of the room. Estimated paths denoted by $\times$ and $\diamond$ represent the accepted and rejected path of the transmitter, respectively.

By solving Eq. (5), we obtain two solutions: $x_{\mathrm{t} 1}$ and $x_{\mathrm{t} 2}$. Then, by substituting them in Eq. (A5), $y_{\mathrm{t} 1}$ and $y_{\mathrm{t} 2}$ are concluded. So, $\left(x_{\mathrm{t} 1}, y_{\mathrm{t} 1}\right)$ is the first solution of transmitter localization and $\left(x_{\mathrm{t} 2}, y_{\mathrm{t} 2}\right)$ is the second one. For a specific receiver placement, there will be only one correct solution for all possible positions of the transmitter.

\section{Simulation study}

\subsection{Description of the use case}

The indoor localization problem will be investigated by observing a room with dimensions of $9.7 \mathrm{~m} \times 4.7 \mathrm{~m} \times 3 \mathrm{~m}$. Two receivers will be placed at the ceiling of the room for logical and practical reasons (this has the purpose of not having a problem with obstacles like furniture or an existence of other objects). Two simulations will be investigated: simulation I is noise-free. Simulation II will be studied under the effect of additive white Gaussian noise (AWGN). For simulation I, we present an irregular source path consisting of 18 discrete positions at $1 \mathrm{~m}$ in height and with a step of approximately $0.5 \mathrm{~m}$ (see Fig. 1). For simulation II, the plane where the transmitter can move (at $1 \mathrm{~m}$ in height) will be discretized in a grid of $40 \times 40$ points. That means the resolution in $x$ direction is about $0.24 \mathrm{~m}$ and in $y$ direction about $0.12 \mathrm{~m}$. For simulation I, the receivers are placed along $y$ axis at the corners of the ceiling with coordinates $\mathrm{S} 1(0,0,3 \mathrm{~m})$ and $\mathrm{S} 2$ $(0,4.7,3 \mathrm{~m})$. In simulation II, the same receiver placement will be used as simulation I but with AWGN.

The radio transceiver AT86RF230 from Atmel (2009) is considered. This transceiver works with a frequency of 2.4 GHz (ZigBee/IEEE802.15.4 applications). The transmitter antenna is simulated by the maximum output power of the transmitter of $P_{\mathrm{t}}=+3 \mathrm{dBm}$ with a gain of $G_{\mathrm{t}}=$ 


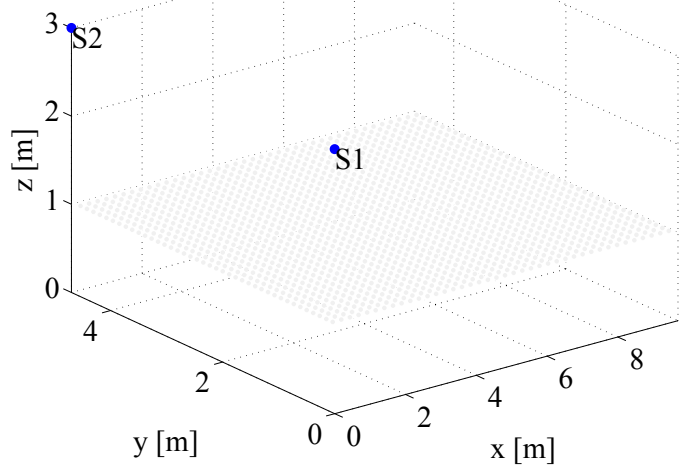

Figure 3. Simulation II: the plane where the transmitter with a height of $1 \mathrm{~m}$ could move is discretized in $40 \times 40$ grid points resulting in a resolution of about $0.24 \mathrm{~m}$ in $x$ direction and about $0.12 \mathrm{~m}$ in $y$ direction. Receivers are placed along the width of the ceiling and at the corners as in simulation I.

$-0.5 \mathrm{dBi}$. The gain of the receiving antenna is set also to $G_{\mathrm{r}}=-0.5 \mathrm{dBi}$.

\subsection{Result and discussion}

\subsubsection{Noise-free simulation}

Simulation I with noise-free data has the purpose of representing the effect of receiver placement on the localization using analytical solution. Solving the quadratic Eq. (5) gives two possible roots for $x$ coordinate of the transmitter position, one inside the boundaries of the specified room and the other one outside the room. The root inside the room $\left(x_{\mathrm{t} 1}\right)$ is chosen automatically as the right one, whereas the other $\left(x_{\mathrm{t} 2}\right)$ has to be rejected. Then, the $y$ coordinate of the transmitter position $y_{\mathrm{t} 1}$ is calculated according to Eq. (A5) given in Appendix A. So, such a placement of receivers in combination with analytical solution method guarantees that the solution $\left(x_{\mathrm{t} 1}, y_{\mathrm{t} 1}\right)$ will always be the right one for all possible transmitter positions in the room, and the second solution $\left(x_{\mathrm{t} 2}\right.$, $y_{\mathrm{t} 2}$ ) will always be outside of the room.

\subsubsection{Simulation with AWGN}

In simulation II, we discretize the plane where the transmitter can move in a grid of $40 \times 40$ points, resulting in a resolution of about $0.24 \mathrm{~m}$ in $x$ direction and about $0.12 \mathrm{~m}$ in $y$ direction (Fig. 3), respectively. Receivers are placed as in simulation I at the corners of the ceiling of the room (Fig. 3). This has the purpose of assessing the robustness of our method by considering the reconstruction error in the whole room. So, the transmitter will be placed each time at one point of the grid and the reconstruction error will be calculated under the AWGN for signal-to-noise ratio $(\mathrm{SNR}=130 \mathrm{~dB})$. Figure 4 shows the reconstruction error at all the points of the grid.

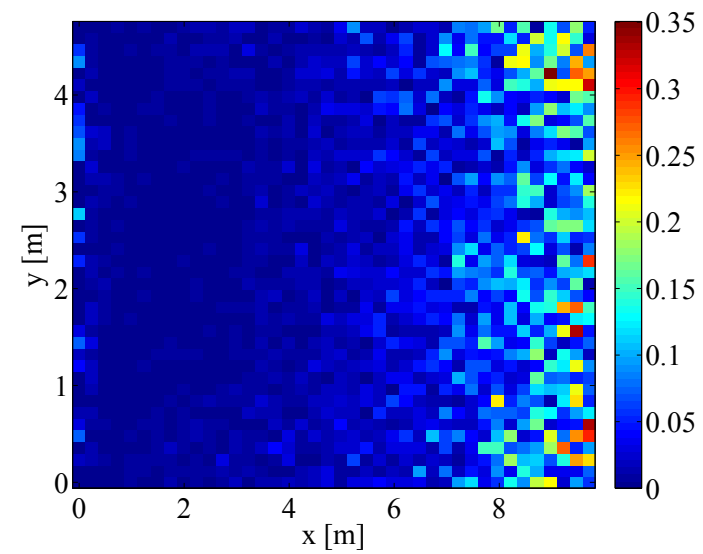

Figure 4. Simulation II: distribution of reconstruction error in the whole room at $1 \mathrm{~m}$ in height. The unit of the color bar is meters. Receivers are placed along the width of the ceiling and at the corners (left side).



Figure 5. Simulation II: distribution of reconstruction error in the whole room at $1 \mathrm{~m}$ in height using the second solution of Eq. (5). Two receivers are placed at the right ceiling's corners and along the width in Fig. 3. The unit of the color bar is meters.

With such an SNR, the mean of reconstruction error for all the points is $0.0340 \mathrm{~m}$. The unit of the color bar is meters. The points far away from receiver positions have higher reconstruction error. If an accuracy like the one in the half of the room near the receivers $\mathrm{S} 1$ and $\mathrm{S} 2$ is desired in the other half, two more receivers can be used at the ceiling's corner (on the right side of Fig. 3). So, the reconstruction error of the whole room will be flipped as seen in Fig. 5. That means, in this case, four receivers will be implemented, and each time just two of them having larger signal strength will be used to reconstruct the source position (two on the left or two on the right). However, in the case of four receivers, a recently introduced scanning method (Warda et al., 2017) can also be applied. 


\section{Conclusions}

In this paper, we presented a new procedure of indoor localization based on the RSSI approach using only two receivers. Our specific arrangement of sensors at the corners of the ceiling of the room solves the ambiguity problem of the roots of the quadratic equation, putting one of the roots outside the boundaries of the room volume. This allows the other root to be a unique solution of the transmitter position. Realistic conditions of the proposed procedure are simulated by adding white Gaussian noise. In that case the reconstruction error averaged over 1600 possible transmitter positions in the room was $3.4 \mathrm{~cm}$, showing the high accuracy of the proposed method.
Data availability. Data used for transceiver AT86RF230 from Amtel (2009) are available at http://www.atmel.com/images/ doc5131.pdf. 


\section{Appendix A}

Let the received power $P_{\mathrm{r}}$ at the position of the receiver $\boldsymbol{R}_{i}$ be related to the transmitted power $P_{\mathrm{t}}$ at the position $\boldsymbol{r}_{j}$ as

$P_{\mathrm{r}}=P_{\mathrm{t}}\left(\frac{\lambda}{4 \pi r}\right)^{2} G_{\mathrm{r}} G_{\mathrm{t}}$.

Because $\lambda$ and $\pi$ are constants and assuming $P_{\mathrm{t}}, G_{\mathrm{t}}$, and $G_{\mathrm{r}}$ as constants too, $P_{\mathrm{r}}$ can be written as

$$
P_{\mathrm{r}}=\frac{c_{1}}{r^{2}} \text {. }
$$

For the first receiver, relation (A3) becomes

$$
P_{\mathrm{r}_{1}}=\frac{c_{1}}{r_{1}^{2}}=\frac{c_{1}}{\left(x_{\mathrm{s} 1}-x_{\mathrm{t}}\right)^{2}+\left(y_{\mathrm{s} 1}-y_{\mathrm{t}}\right)^{2}+\left(z_{\mathrm{s} 1}-z_{\mathrm{t}}\right)^{2}},
$$

and for the second receiver it becomes

$$
P_{\mathrm{r}_{2}}=\frac{c_{1}}{r_{2}^{2}}=\frac{c_{1}}{\left(x_{\mathrm{s} 2}-x_{\mathrm{t}}\right)^{2}+\left(y_{\mathrm{s} 2}-y_{\mathrm{t}}\right)^{2}+\left(z_{\mathrm{s} 2}-z_{\mathrm{t}}\right)^{2}} .
$$

Because the coordinates of receivers are known $\left(x_{\mathrm{s} 1}, y_{\mathrm{s} 1}, z_{\mathrm{s} 1}\right.$ and $\left.x_{\mathrm{s} 2}, y_{\mathrm{s} 2}, z_{\mathrm{s} 2}\right)$ and assuming the transceiver at the known height $z_{\mathrm{t}}$, the only unknowns in Eqs. (A3) and (A4) are $x_{\mathrm{t}}$ and $y_{\mathrm{t}}$. So, Eqs. (A3) and (A4) are functions of $x_{\mathrm{s} 1}, y_{\mathrm{s} 1}, x_{\mathrm{s} 2}, y_{\mathrm{s} 2}$ and $x_{\mathrm{t}}, y_{\mathrm{t}}$. By subtracting Eq. (A4) from Eq. (A3) and solving this according to $y_{\mathrm{t}}$, we get

$$
y_{\mathrm{t}}=\frac{c_{3}-2 x_{\mathrm{t}}\left(x_{\mathrm{s} 2}-x_{\mathrm{s} 1}\right)}{2\left(y_{\mathrm{s} 2}-y_{\mathrm{s} 1}\right)},
$$

where

$c_{3}=c_{2}-x_{\mathrm{s} 1}^{2}-y_{\mathrm{s} 1}^{2}+x_{\mathrm{s} 2}^{2}+y_{\mathrm{s} 2}^{2}$

and

$c_{2}=\frac{c_{1}}{P_{\mathrm{r}_{1}}}-\left(z_{\mathrm{s} 1}-z_{\mathrm{t}}\right)^{2}-\frac{c_{1}}{P_{\mathrm{r}_{2}}}+\left(z_{\mathrm{s} 2}-z_{\mathrm{t}}\right)^{2}$.

Then, substituting Eq. (A5) in Eq. (A3), we get an equation with unknown $x_{\mathrm{t}}$. This equation is a quadratic one, and it is a function of the transmitter's coordinate $x_{\mathrm{t}}$ :

$a x_{\mathrm{t}}^{2}+b x_{\mathrm{t}}+c=0$,

where $a, b$, and $c$ are constants related to the receiver's coordinates and the height of the transmitter.

$$
\begin{aligned}
a & =1+\frac{\left(x_{\mathrm{s} 2}-x_{\mathrm{s} 1}\right)^{2}}{\left(y_{\mathrm{s} 2}-y_{\mathrm{s} 1}\right)^{2}} \\
b & =\frac{2 y_{\mathrm{s} 1}\left(x_{\mathrm{s} 2}-x_{\mathrm{s} 1}\right)}{\left(y_{\mathrm{s} 2}-y_{\mathrm{s} 1}\right)}-\frac{c_{3}\left(x_{\mathrm{s} 2}-x_{\mathrm{s} 1}\right)}{\left(y_{\mathrm{s} 2}-y_{\mathrm{s} 1}\right)^{2}}-2 x_{\mathrm{s} 1} \\
c & =\left[\frac{c_{1}}{P_{\mathrm{r}_{1}}}-\left(z_{\mathrm{s} 1}-z_{\mathrm{t}}\right)^{2}-x_{\mathrm{s} 1}^{2}-y_{\mathrm{s} 1}^{2}\right. \\
& \left.+\frac{y_{\mathrm{s} 1} c_{3}}{\left(y_{\mathrm{s} 2}-y_{\mathrm{s} 1}\right)}-\frac{c_{3}^{2}}{4\left(y_{\mathrm{s} 2}-y_{\mathrm{s} 1}\right)^{2}}\right]
\end{aligned}
$$


Competing interests. The authors declare that they have no conflict of interest.

Edited by: Rosario Morello

Reviewed by: two anonymous referees

\section{References}

Atmel: AT86RF230, available at: http://www.atmel.com/images/ doc5131.pdf (last access: 28 March 2017), 2009.

Bishop, A. N., Fidan, B., Anderson, B. D., Doğançay, K., and Pathirana, P. N.: Optimality analysis of sensortarget localization geometries, Automatica, 46, 479-492, https://doi.org/10.1016/j.automatica.2009.12.003, 2010.

Fang, B. T.: Simple solutions for hyperbolic and related position fixes, IEEE T. Aero. Elec. Sys., 26, 748-753, 1990.

Farid, Z., Nordin, R., and Ismail, M.: Recent advances in wireless indoor localization techniques and system, Journal of Computer Networks and Communications, 2013, 185138, https://doi.org/10.1155/2013/185138, 2013.
Garg, V. K.: Wireless Communications and Networking, Elsevier Morgan Kaufmann, San Francisco, USA, 2007.

Han, G., Choi, D., and Lim, W.: A novel reference node selection algorithm based on trilateration for indoor sensor networks, in: 7th IEEE International Conference on Computer and Information Technology (CIT 2007), 1003-1008, 16-19 October 2007, AizuWakamatsu, Fukushima, Japan, 2007.

Linde, H.: On aspects of indoor localization, $\mathrm{PhD}$ thesis, Universität Dortmund, 2006.

Warda, A., Petković, B., and Toepfer, H.: Scanning method for indoor localization using the RSSI approach, J. Sens. Sens. Syst., 6, 247-251, https://doi.org/10.5194/jsss-6-247-2017, 2017.

Yassin, A., Nasser, Y., Awad, M., Al-Dubai, A., Liu, R., Yuen, C., and Raulefs, R.: Recent advances in Indoor Localization: A Survey on Theoretical Approaches and Applications, IEEE Commun. Surv. Tut., 19, 1327-1346, 2016.

Zhang, Y., Yang, L. T., and Chen, J.: RFID and Sensor Networks: Architectures, Protocols, Security, and Integrations, CRC Press, Taylor \& Francis Group, Boca Raton, Florida, USA, 2009. 